Running Head: $\quad$ PERSONALITY OF YOUNG CHESS PLAYERS

Personality Profiles of Young Chess Players 


\title{
PERSONALITY OF YOUNG CHESS PLAYERS
}

\begin{abstract}
Although the game of chess has often featured in psychological research, we know very little about people who play chess, especially about children who take up chess as a hobby. This study presents the personality profiles as measured with the Big Five model (BFQ-C; Barbaranelli et al., 2003) of 219 young children who play chess and 50 of their peers who do not. Children who score higher on Intellect/openness and Energy/extraversion are more likely to play chess while children who score higher on Agreeableness are less likely to be attracted to chess. Boys with higher scores on Agreeableness are less likely to take up chess than boys with lower scores. Considering that girls score higher on Agreeableness, this factor may provide one of the possible reasons why more boys are interested in chess. Although none of the Big Five factors were associated with self-reported skill level, a sub-sample of 25 elite players had significantly higher scores on Intellect/openness than their weaker chess playing peers.
\end{abstract}

Key words: Chess, Personality, Big Five Model, Children, Gender differences 


\section{PERSONALITY OF YOUNG CHESS PLAYERS}

\section{Personality Profiles of Young Chess Players}

The game of chess has been frequently used in psychological research. Some have even claimed that it plays a pivotal role in cognitive psychology comparable to that of Drosophila in genetics (Simon \& Chase, 1973). Given the prominent status of chess in psychology, it is surprising that we know little about (the personalities of) the people who perform this activity. Even more surprising is that we know virtually nothing about (the personality characteristics of) children who decide to take up chess as a hobby. With this study we try to close this gap by reporting the personality profiles, as measured with the Big Five model, of children who play chess and those who do not. We also investigate the impact that personality characteristics have on the different interest of boys and girls for chess as well as on chess skill.

It is not unusual for a chess game to take several hours. In that time the two players are alone, fighting each other with their knowledge and will-power. So it is not surprising that Kelly (1985), using the Myers-Briggs Type Indicator, found that chess players are more introverted and intuitive than the general population. In addition, stronger players also tend to be more intuitive than weaker ones.

Chess is an adversarial game where one has to take into account the opponent's intentions and not just focus on one's own plans. Chess is also a game where just a small mistake can ruin the efforts of the previous long hours. Hence, players should be more suspicious and orderly than non-players. That is exactly what Avni, Kipper, and Fox (1987) demonstrated - chess players scored higher than non-players on the measures of orderliness and unconventional thinking in the Minnesota Multiphasic Personality Inventory. In addition, it was found that more competitive players, as measured by the number of games played, were more suspicious than non-players.

The competitive side of chess has not escaped the attention of researchers. Mazur, Booth, and Dabbs (1992) found that testosterone level, usually linked to aggressiveness (see 


\section{PERSONALITY OF YOUNG CHESS PLAYERS}

Mazur \& Booth, 1998), tends to be higher in winners than losers and, with some players, to rise just before their games. Similarly, Joireman, Fick, and Anderson (2002) showed that sensation seeking discriminates between college students who tried out chess and those who never played chess, and predicts frequency of playing.

A common observation, potentially related to personality factors, is that there are clearly more men playing chess than women. For every woman good enough to be rated by the International Chess Federation (FIDE), there are fourteen men (Howard, 2005). While there are several possible reasons why men play better chess than women (see Charness \& Gerchak, 1996; Howard, 2005), there have been hardly any suggestions as to why more men play chess. While it is plausible that factors such as intelligence, motivation, and practice influence how good at chess people will become, it seems reasonable to assume that personality factors may influence people's preferences for hobbies (e.g., Avni et al., 1987).

This little evidence we have about people who play chess was all obtained in adult samples. Long is the path to being an active adult chess player and inevitably some people who learn to play chess will stop at some point. We are thus left to wonder who are the people, or more precisely, children, who start with chess in the first place. Why there is such a large discrepancy in the numbers of girls and boys that take up chess? It is also not known who are the children who will find chess so engaging that they will spend more time practising than their peers and become skilled chess players. In this study we investigated the personality profiles of primary school children who play chess and those who do not with the popular Big Five (BF) model (Costa \& McCrae, 1988). Until recently, self-reported measures of the BF model in early and middle childhood have been rarely used. The reason was that researchers believed that children at that age are not able to give reliable self-reports (see Harter, 1998). This view has been recently challenged (e.g., Shiner, 1998) and meanwhile there are indications that self-reports are not only a valid method of measuring personality characteristics in late 
childhood (e.g., Barbaranelli, Capara, Rabasca, \& Pastorelli, 2003), but also that they may provide the best way for measurement very early in life (Ablow et. al., 1999; Marsh, Ellis, \& Craven, 2003). For example, Measelle and colleagues (Measelle et al., 2005) recently followed five year olds for two years and demonstrated that the BF factors were evident, stable, and replicated throughout this period of life.

We applied the Big Five Questionnaire for Children (BFQ-C; Barbaranelli et al., 2003), which measures Energy/extraversion, Agreeableness, Conscientiousness, Emotional instability, and Intellect/openness, on primary school children aged eight to eleven. Our main goal was to find out what are the personality characteristics of children who decide to take up chess, as well as to see whether personality factors can differentiate between strong and weak players. We also wanted to see whether personality factors could shed some light on the issue of the large discrepancies in the participation rates of girls and boys. Based on previous results with adults (e.g., Avni et al. 1987; Joireman et al., 2002; Kelly, 1985), we hypothesised that children who play chess would score more highly on Conscientiousness but less highly on Energy/extraversion than children who do not play chess. Given that chess is often perceived as an intellectual endeavour, we also hypothesised that Intellect/openness will differentiate between children who take up chess and those who do not. The same personality factors could be expected to differentiate between strong and weak child chess players.

Since women score higher on Emotional instability and Agreeableness (Costa, Terracciano, \& McCrae, 2001; Goodwin \& Gotlib, 2004; Rubinstein, 2005), two factors previously not shown to be associated with chess skill, it is difficult to have clear-cut predictions as to how these factors are related to gender differences in chess skill. On the other hand, chess has a competitive side where players encounter constant conflicts and confrontations (e.g., Mazur et al., 1992), which may be less appealing to children who score 
more on Agreeableness. Consequently, it is possible that Agreeableness provides clues about the differences in the number of girls and boys who take up chess as a hobby.

Method

\section{Participants}

Two hundred and sixty-nine primary school children (mean age $=10.1$ years, $S D=1.2$ ) from four schools in Oxfordshire, UK, participated in the study. Two thirds were male; there were 45 eight year olds, 61 nine year olds, 70 ten year olds, and 93 eleven year olds. All schools had chess club sessions at least once a week and actively participated in regional primary school competitions. So all the children had a chance to take part in chess activities.

\section{Instrument and procedure}

The BFQ-Q (Barbaranelli et al., 2003) was applied. There are 65 items in the questionnaire which are supposed to measure the traits of Extraversion/energy (e.g., activity, enthusiasm, assertiveness and self-confidence), Agreeableness (sensitivity towards others and their needs), Conscientiousness (dependability, orderliness, precision, fulfilment of commitments), Emotional instability (feelings of anxiety, depression, discontent, and anger), and Intellect/openness (intellect in school domain, broadness of cultural interests, fantasy, creativity, and interest in other people). Each factor is measured with 13 items. Previous research (Barbaranelli et al., 2003; Muris, Meesters, \& Diederen, 2005) shows that the BFQ-C has satisfactory internal structure and is a valid instrument for the measurement of the big five traits. Before using the BFQ-C, the questionnaire was amended with the help of several primary school teachers so that it corresponded to the English language used in the UK. The questionnaire was piloted individually on a number of primary school children in the third and fourth grade (eight and nine years old) in order to ensure that even the youngest children in our sample could understand the words used in the questionnaire. Another change to accommodate the young children in our sample was the use of a three point Likert type scale $(1=$ Almost 


\section{PERSONALITY OF YOUNG CHESS PLAYERS}

never, $2=$ Sometimes, and $3=$ Almost always) instead of the usual 5 point scale. The final score of each of the five traits is obtained by adding the individual item scores (the maximum score being 39).

In addition to the BFC-Q questionnaire, two questions concerning chess playing were asked at the beginning of the questionnaire. The first question asked whether the child knew how to play chess (knew the rules) while the second question asked how good at chess they were in comparison with their peers. The first question could be answered with yes and no while the second employed a 5 point Likert scale - from $1=$ very bad to 5 very good. The children who did not play chess did not answer the second question about their skill level and were not included in the analysis performed on that question. The questionnaire was administered in groups during the school hours by the first author and the teacher of the class where the testing took place. Great care was taken to ensure that all children understood that it was not a test and that their teachers would not be able to inspect their answers.

\section{Results}

Principal component factor analysis with oblimin rotation, the same method used in previous studies with the BFQ-C (Barbaranelli et al., 2003; Muris et al., 2005), yielded twenty factors with eigenvalues higher than one $(9.25,3.78,3.12,2.46,2.06,1.77,1.63,1.54$, and so on). We extracted the hypothesized five factors which accounted for $32 \%$ of the total variance. The factors of Agreeableness, Conscientiousness, and Emotional instability had the expected structure where almost all theoretically predefined items unambiguously loaded on one single factor. (The item loadings can be obtained by writing to the authors.) The same cannot be claimed for the factors of Energy/extraversion and Intellect/openness, where only the majority of the items loaded on the theoretical factors. While it was difficult to categorize the items which loaded on Energy/extraversion and those which did not, in the case of Intellect/openness, it seemed that the openness component did not load convincingly. The Intellect component, on 
the other hand, displayed the expected pattern of results. Consequently, the internal consistency of the created five scales as measured by Cronbach's alpha was the highest for Agreeableness, Emotional instability, and Conscientiousness (.81, .79, and .78, respectively) and lowest for Energy/extraversion and Intellect/openness (.68 and .72, respectively).

It is possible that the young children participating in this study have not yet fully developed the traits of Openness and Extraversion. This possibility seems to gain credibility in the light of the results of Measelle et al. (2005) who found that the factors of Extraversion and Intellect, the same unclear factors in our study, were not as internally consistent in children between 5 and 7 years old as they were in a sample of college students. In contrast, Agreeableness, Conscientiousness, and Emotional instability were as internally consistent in children as they were in students. However, even when we dropped the two youngest groups (eight and nine year olds) from the factor analysis, the results remained basically the same, which implies that the problems may lie in the way the items were phrased.

Another plausible reason may be the restriction of the answer range (from 1-5 to 1-3) and slight alterations of the items to adjust them to the participants' culture. It is also possible that the instrument itself captured these two traits unsatisfactorily given that previous studies by Muris et al. (2005), and partly Barbaranelli et al. (2003), indicated similar problems. The BFQ$\mathrm{C}$ seems to be a relatively valid and reliable instrument for the measurement of the BF model but may need some additional work on the factors of Energy/extraversion and Intellect/openness.

Descriptive statistics and inter-correlations for all variables featured in the study can be seen in Table 1. The last two columns in Table 1 show the scores of children who can play chess (players) and those who cannot (non-players). Chess players were predominantly boys, significantly older than non-players, had significantly higher scores on Energy/extraversion and Intellect/openness, and significantly lower scores on Agreeableness than non-players. 
Table 1

We performed a logistic regression to investigate the personality characteristics of children who play chess. We used all five personality factors together with gender and age as the predictors of chess involvement. Given a number of significant inter-correlations (see Table 1), we felt that this approach would enable us to see how individual predictors contribute towards discriminating players and non-players when they act together. The logistic regression confirmed the results obtained when the individual predictors were analysed separately. Children who played chess were more likely to be boys $(B=.77$, Wald (the test for the significance of the logistic regression coefficient obtained squaring the ratio of the coefficient and its standard error $)=4.2, p<.05)$, were older $(B=.38$, Wald $=6.5, p<.05)$, had higher scores on Extraversion $(B=.15$, Wald $=5.1, p<.05)$ and Intellect/openness $(B=.23$, Wald $=$ $12.3, p<.01)$ but lower scores on Agreeableness $(B=-.18$, Wald $=8.6, p<.01)$.

Table 1 indicates that the proportion of the girls who take up chess $(68 \%)$ is smaller than the proportion of the boys $\left(88 \% ; \chi^{2}(1, N=269)=14.3 ; p<.01\right)$. In addition, Agreeableness was the only personality factor associated both with gender and with whether or not children played chess - boys and players scored less on Agreeableness than girls and non-players. In order to check whether the scores on Agreeableness influenced the choice of chess as a hobby without regard to gender, we analysed the sub-sample of boys. When only boys were included in the analysis, the association between playing chess and the score on Agreeableness was still significant $(r(182)=-.14 ; p<.05)$ and there was also a significant difference between the agreeableness scores of boys who play chess $(M=29.8, S D=4.2)$ and those who do not $(M=$ 31.6, $S D=3.6 ; t(182)=2.1 ; p<.05)$. Hence, the association between Agreeableness and chess 


\section{PERSONALITY OF YOUNG CHESS PLAYERS}

playing seems to be a genuine consequence of the scores on Agreeableness and not the fact that there are more girls, who on average score more on Agreeableness, among the children who do not play chess.

Gender and the personality factors did not significantly predict perceived chess skill when we performed a regression analysis with all factors included. This is not surprising considering that there were no significant correlations between self-reported chess skill and personality factors (see Table 1). Splitting the sample into two groups, comprising the children who reported that they are good or very good at chess, and those who assessed themselves as less able, did not alter the results - none of the personality factors discriminated between the two groups.

It is possible that self-reported chess skill is a biased measure of chess skill especially considering that children had to indicate their skill level in comparison with their peers. We investigated this possibility with an elite sub-sample of young chess players for which we had an objective estimate of skill. The elite sub-sample comprised 25 players who were playing regularly in chess competitions and possessed a chess rating. A player's chess rating is based purely on their results against other players and is thus an objective measure of chess skill. Some of the children in the elite sub-sample were selected to represent England in international competitions on numerous occasions. Their average rating on the British Chess Federation (BCF) scale was $71(S D=22) .{ }^{1}$ When converted to the International FIDE rating, the average rating was $1605(S D=108)$. The highest rating was 1835 (more than $1.5 \mathrm{SD}$ above the mean) while the lowest was 1380 (half a SD below the average player). Given that the rating of the average player (including both adults and children) is 1500 , our sample was truly exceptional for children.

Table 2 presents the personality profiles of elite chess players and chess players (the profiles of non-chess players are presented for the sake of completeness). Elite chess players 


\section{PERSONALITY OF YOUNG CHESS PLAYERS}

tended to be more emotionally stable, more energetic/extraverted, and more conscientious than all chess players, although the only significant difference between the two groups was in the extent of Intellect/openness. Elite chess players turned out to be significantly more open to new experiences and more curious than their less (chess) able peers. Elite chess players, who were exclusively male, were also older.

\section{Table 2}

A logistic regression with the personality factors and age as the predictors indicated that age $(B=1.25$, Wald $=17.9, p<.01)$ and partly Intellect/openness $(B=.15$, Wald $=3.2, p<$ .10) were significant predictors of the elite players. It should be noted that within this small and elite sub-sample none of the personality factors were significantly associated with the rating when age was controlled for (the highest correlation was around .30 for Conscientousness and Agreeableness, while there was no association with Intellect/openness). We also acknowledge that the factors of Energy/extraversion and Intellect/openness, which distinguished among elite players, players and non-players, were the least reliably measured by the BFC-Q. Although this casts a shadow of doubt on our findings and conclusions (see Discussion), the pattern of the results did not change when we included only the reliable items in the final score of the Energy/extraversion and Intellect/openness scales.

\section{Discussion}

The present study provides, for the first time, a glimpse at the personality of children who are likely to take up chess. Children who are less sensitive towards others, more prone to arguing and less to avoiding conflicts (Agreeableness), more energetic (Energy/extraversion) and more open to new experience (Intellect/openness) are more likely to be attracted to the game of chess. Given the competitive and aggressive side of chess (e.g., Mazur et al., 1992), 


\section{PERSONALITY OF YOUNG CHESS PLAYERS}

the finding that children who score lower on Agreeableness are more likely to start chess seems reasonable. Chess is a game of constant conflicts where each side tries to overwhelm and out hustle the other. This aggressive component may also be one of the reasons for the gender differences in the participation rates. The competitive component of chess may be more appealing to boys than girls, even right at the start.

In our study, for example, boys scored lower on Agreeableness than girls. The same finding about the gender differences on Agreeableness has been repeatedly obtained in adult samples (e.g., Costa, Terracciano, \& McCrae, 2001; Goodwin \& Gotlib, 2004; Rubinstein, 2005). It is indicative for the role of Agreeableness in choosing chess that even among the boys, higher agreeableness scores were found among the children who did not play chess.

Agreeableness may indeed go a long way in explaining why more boys than girls take up chess as a hobby.

Similarly, a predictable result was that children who are prone to intellectual activities, generally curious, and open to new experiences are likely to find chess interesting. Chess has the aura of an activity where one has to overcome obstacles with intellectual and not physical power. It is possible, however, that children who are more open to new experience are in general more likely to try out more different activities than their peers who are less open to new experiences and less intellectual. A slightly more surprising result, at least at first sight, is that the more energetic and extraverted children are more likely to play chess. This seems to be in contradiction with the finding that adult chess players are more introverted than the general population (Avni et al., 1987). Extraverted and energetic children are, however, in general more likely to try out activities than their less extraverted peers. Hence it should not be overly surprising to find that more extraverted children are more likely to have played chess. Finally, this does not mean that they will continue to play chess nor that they will invest more time in it than their less extraverted peers. 


\section{PERSONALITY OF YOUNG CHESS PLAYERS}

The second set of analyses confirms this speculation. Extraversion, together with all other factors, did not predict how well the children in this study perceived their skill level. This pattern of results may indicate that personality plays only an important role in chess involvement and not in determining the extent of chess skill. On the other hand, it is possible that, while personality does not predict self-reported skill level, it plays a role when objective measures of chess skill are applied. The subsequent analysis with the elite sub-sample of young chess players points to the latter possibility. When an objective measure of chess skill, such as rating, was taken into account, Intellect/openness did discriminate between the elite young chess players and weaker players. The elite children players were more curious, had broader intellectual and cultural interests, and were more accomplished in school than weaker players. Since Intellect/openness is positively associated with intelligence (e.g., Austin, Deary, \& Gibson, 1997; Harris, 2004), we could infer that intelligence is an important aspect of chess skill in young children. This fits well with a couple of studies which demonstrate that children who are good chess players score higher on intelligence tests than their peers who are less accomplished players (e.g., Horgan \& Morgan, 1990; Frydman \& Lynn, 1992). But it would be premature, if not wrong, to extrapolate from these results on children that intelligence, or Intellect/openness, plays an important role in chess in general as some authors do (e.g., Howard, 2005; but see Bilalić \& McLeod, 2006). This would be particularly dubious when even after a century of investigation not a single study with adult chess players has managed to establish a link between chess skill and intelligence (for a review see Gobet, de Voogt, \& Retschitzki, 2004). Finally, Intellect/openness had little predictive power among the elite subsample when the other factors were controlled for.

The discussion about intelligence and chess leads us to acknowledge the limits of our study. While we showed that personality profiles of elite young chess players, children who play chess and those who do not are indeed different, we did not include other factors of 


\section{PERSONALITY OF YOUNG CHESS PLAYERS}

interest (e.g., motivation, intelligence) and investigate how they interact. This may not be so crucial for the differentiation between children who play and those who do not play chess, but it may prove important for the differentiation between the elite and less good young players. It would be incorrect, in our opinion, to claim that Intellect/openness explains well why children became elite players at their age. It is possible that other characteristics, such as the amount of time spent playing chess and motivation, as well as intelligence, have a more direct influence on chess skill than personality. Nevertheless, the role of personality characteristics in chess development seems to be assured. It remains, however, to be seen whether its influence is of a direct nature or more of an indirect nature - through the influence of other factors such as motivation and practice. 


\section{PERSONALITY OF YOUNG CHESS PLAYERS}

\section{References}

Ablow, J. C., Measelle, J. R., Kraemer, H. C., Harrington, R., Luby, J., Smider, N., et al. (1999). The MacArthur Three-City Outcome Study: Evaluating multi-informant measures of young children's symptomatology. Journal of the American Academy of Child and Adolescent Psychiatry, 38, 1580-1590.

Austin, E. J., Deary, I. J., \& Gibson, G. J. (1997). Relationships between ability and personality: Three hypotheses tested. Intelligence, 25, 49-70.

Avni, A., Kipper, D.A., \& Fox, S. (1987). Personality and leisure activities: An illustration with chess players. Personality and Individual Differences, 8, 715-719.

Barbaranelli, C., Caprara, G. V., Rabasca, A., \& Pastorelli, C. (2003). A questionnaire for measuring the Big Five in late childhood. Personality and Individual Differences, 34, $645-664$.

Bilalić, M. \& McLeod, P. (2006). How intellectual is chess? - A reply to Howard. Journal of Biosocial Science, 38, 419-421.

Charness, N., \& Gerchak, Y. (1996). Participation rates and maximal performance: A log-linear explanation for group differences, such as Russian and male dominance in chess. Psychological Science, 7, 46-51.

Costa, P. T. Jr., \& McCrae, R. R. (1988). From catalog to classification: Murray's needs and the five factor model. Journal of Personality and Social Psychology, 55, 258-265.

Costa, P. T., Jr, Terracciano, A., \& McCrae, R. R. (2001). Gender differences in personality traits across cultures: Robust and surprising findings. Journal of Personality and Social Psychology, 81, 322-331.

Frydman, M. \& Lynn, R. (1992). The general intelligence and spatial abilities of gifted young Belgian players. British Journal of Psychology, 83, 233-235. 


\section{PERSONALITY OF YOUNG CHESS PLAYERS}

Gobet, F., de Voogt, A. \& Retschitzki, J. (2004). Moves in mind - The psychology of board games. Hove, UK: Psychology Press.

Goodwin, R.D. \& Gotlib, I. H. (2004). Gender differences in depression: The role of personality factors. Psychiatry Research, 126, 135-142.

Harris, J. A. (2004). Measured intelligence, achievement, openness to experience, and creativity. Personality and Individual Differences, 36, 913-929.

Harter, S. (1998). The development of self-representations. In W. Damon (Series Ed.) \& N. Eisenberg (Vol. Ed.), Handbook of child psychology: Vol. 3. Social, emotional, and personality development (5th ed., pp. 553-617). New York: Wiley.

Horgan, D. E. \& Morgan, D. (1990). Chess expertise in children. Applied Cognitive Psychology, 4, 109-128.

Howard, R. W. (2005). Are gender differences in high achievement disappearing? A test in one intellectual domain. Journal of Biosocial Science, 37, 371-380.

Joireman, J.A., Fick, C.S., \& Anderson, J.W. (2002). Sensation seeking and involvement in chess. Personality and Individual Differences, 32, 509-515.

Kelly, E.J. (1985). The personality of chessplayers. Journal of Personality Assessment, 49, 282284.

Marsh, H. W., Ellis, L. A., \& Craven, R. G. (2003). How do preschool children feel about themselves? Unraveling measurement and multidimensional self-concept structure. Developmental Psychology, 38, 376-393.

Mazur, A. \& Booth, A. (1998). Testosterone and dominance in men. Behavioral and Brain Sciences, 21, 353-397.

Mazur, A., Booth, A., \& Dabbs, J.M. (1992). Testosterone and chess competition. Social Psychology Quartely, 55, 70-77. 


\section{PERSONALITY OF YOUNG CHESS PLAYERS}

Measelle, J. R., John, P. O., Ablow, C. J., Cowan, A. P., \& Cowan, P. C. (2005). Can children provide coherent, stable, and valid self-reports on the Big Five dimensions? A longitudinal study from ages 5 to 7. Journal of Personality and Social Psychology, 89, 90-106.

Muris, P., Meesters, C. \& Diederen, R. (2005). Psychometric properties of the Big Five Questionnaire for Children (BFQ-C) in a Dutch sample of young adolescents. Personality and Individual Differences, 38, 1757-1769.

Rubinstein, G. (2005). The big five among male and female students of different faculties. Personality and Individual Differences, 38, 1495-1503.

Shiner, R. L. (1998). How shall we speak of children's personalities in middle childhood?: A preliminary taxonomy. Psychological Bulletin, 124, 308-332.

Simon, H.A., \& Chase, W.G. (1973). Skill in chess. American Scientist, 61, 393-403. 


\section{PERSONALITY OF YOUNG CHESS PLAYERS}

\section{Footnote}

${ }^{1}$ There is an international FIDE rating (also called ELO rating) but usually national federations have their own rating (e.g., BCF). The ELO rating has a theoretical mean of 1500 and a standard deviation of 200 . Thus all players over 1500 can be said to be fairly skilled; masters normally have ratings over 2200, and grandmasters over 2500 . The formula for converting BCF into ELO is: $\left(\mathrm{BCF}^{*} 5\right)+1250$. 


\section{PERSONALITY OF YOUNG CHESS PLAYERS}

Table 1

Descriptive statistics, intercorrelations, and profiles of children who play chess (Players; $n=219)$ and those who do not (Non-players; $n=50)$

\begin{tabular}{|c|c|c|c|c|c|c|c|c|c|c|c|}
\hline & & & & & & & & & & Players & Non-players \\
\hline & 1 & 2 & 3 & 4 & 5 & 6 & 7 & 8 & 9 & $\mathrm{M}(\mathrm{SD})$ & $\mathrm{M}(\mathrm{SD})$ \\
\hline (1) Gender (2 = girls) & -- & -.01 & .03 & $.25^{* *}$ & .07 & $.16^{* *}$ & -.08 & -.11 & $-.24 * *$ & $26 \%$ girls & $54 \%$ girls \\
\hline (2) Age & & -- & .10 & -.10 & -.09 & .04 & .01 & $.19 * *$ & $.19 *$ & $10.2(1.2)^{* *}$ & $9.6(1.3)$ \\
\hline (3) Energy/extraversion & & & -- & $.34 * *$ & $.27 * *$ & $-.20 * *$ & $.32 * *$ & $.16^{*}$ & .11 & $32.3(3.2)^{*}$ & $31(3)$ \\
\hline (4) Agreeableness & & & & -- & $.62 * *$ & $-.29 * *$ & $.38 * *$ & $-.15 *$ & .05 & $30.5(4.3)^{*}$ & $32.1(3.8)$ \\
\hline (5) Conscientiousness & & & & & -- & $-.34 * *$ & $.45^{* *}$ & -.05 & .02 & $28.7(4.3)$ & $29.2(4.4)$ \\
\hline (6) Emotional instability & & & & & & -- & $-.17 * *$ & -.09 & .00 & $24.5(4.4)$ & $25.5(5)$ \\
\hline (7) Intellect/openness & & & & & & & -- & $.19 * *$ & .05 & $31(3.3)^{* *}$ & $29.2(3.9)$ \\
\hline (8) Playing chess (yes/no) & & & & & & & & -- & -- & & \\
\hline (9) Chess skill (1-5) & & & & & & & & & -- & & \\
\hline$M$ & 1.32 & 10.10 & 32.06 & 30.78 & 28.81 & 24.68 & 30.64 & $81 \%$ yes & 2.76 & & \\
\hline$S D$ & 0.47 & 1.20 & 3.19 & 4.22 & 4.28 & 4.50 & 3.47 & & 1.53 & & \\
\hline
\end{tabular}

Note. ${ }^{*} p<.05, * * p<.01$ 
PERSONALITY OF YOUNG CHESS PLAYERS

Table 2

Personality profiles of the elite sub-sample $(n=25)$, weaker players $(n=194)$, and non-players $(n=50)$

\begin{tabular}{lccc}
\hline & Elite players & Players & Non-players \\
\hline Extraversion & $\mathrm{M}(\mathrm{SD})$ & $\mathrm{M}(\mathrm{SD})$ & $\mathrm{M}(\mathrm{SD})$ \\
Agreeableness & $30(3.1)$ & $32.2(3.2)$ & $31(3)$ \\
Conscientiousness & $29.8(4.2)$ & $28.6(4.3)$ & $29.2(4.4)$ \\
Instability & $24.8(5.1)$ & $24.5(4.3)$ & $25.5(4)$ \\
Openness* & $32.7(3.2)$ & $30.8(3.3)$ & $29.2(3.9)$ \\
Age* & $11.2(1.2)$ & $10.1(1.1)$ & $9.6(1.3)$ \\
Gender (girls) & $0 \%$ & $29 \%$ & $54 \%$ \\
\hline Note. ${ }^{*} p<.01$ & & &
\end{tabular}

